\title{
Advancements in the management of thoracic aortic dissection
}

Aortic dissection, a tear in the intima of the aorta in which new blood flow into a false lumen is established, was first described as such by German anatomist Daniel Sennert in the 16th century (1). Since the death of George II, King of England in 1760 and the autopsy performed by Frank Nicholls we have known of the grave dangers of acute aortic dissection. Centuries of hard work and innovation within the global medical community has helped to increase understanding of the predisposing factors, etiology, management and treatment of dissection, yet it continues to be a vexing problem with high morbidity and mortality. In this focused series of fournal of Visualized Surgery (FOVS), experts from prominent aortic centers from around the United States will outline the surgical management of thoracic aortic dissection from the aortic root to the distal thoracic aorta. The development of endovascular technology has been instrumental in the treatment of thoracic aortic dissection but has not yet been able to transform aortic dissection into a disease completely able to be treated with endovascular approaches.

The treatment for thoracic aortic dissection depends on several simple principles that must be addressed with any strategy for repair, be it endovascular or open. These principles include accurate and timely diagnosis, immediate initiation of antiimpulse therapy and blood pressure control, restoration of true lumen flow, exclusion of the intimal tear and false lumen and postoperative surveillance of any residual dissection. This series will provide several examples of therapeutic strategies for different sections of the thoracic aorta, all of which will address the basic principles of management stated previously.

Acute dissection of the aortic root, ascending aorta or aortic arch is a particularly dangerous condition with a reported mortality rate of $1 \%$ per hour initially, $50 \%$ mortality after 72 hours and $80 \%$ mortality within 2 weeks of diagnosis if untreated $(2,3)$. The presence of a type A aortic dissection (TAAD) is an indication for repair in all but the highest risk patients. Age alone should not be a contraindication to repair. Treatment of TAAD begins with diagnosis and it is important for patients presenting with hypertension and classic symptoms of tearing chest pain to be properly evaluated on presentation with appropriate diagnostic studies with arterial phase contrast computed tomography (CT) being the diagnostic study of choice. It is not uncommon for patients to present with chest pain and dyspnea and undergo workup for pulmonary embolism with a contrast CT scan in the pulmonary arterial phase, which, if convincing for the diagnosis does not need to be repeated in arterial phase to avoid a second contrast bolus. Blood pressure control with short acting beta blockers should be initiated and continued until the patient can be taken to the operating room.

The mainstay of treatment for TAAD has been surgical repair with replacement of the affected segments of the aorta and its branches $(4,5)$. Endovascular repair of the ascending aorta has not become standard of care but has been attempted and successfully performed in certain high-risk groups with reasonable outcomes. In this series of $7 \mathrm{OVS}$ the group from the University of Maryland present a review of the literature and their own institutional results of endovascular repair of TAAD. Endovascular repair presents many challenges in the ascending aorta regarding management of the aortic valve, coronary ostia and arch branch vessels, however, the review is informative and their results are impressive in their select group of patients.

Current standard of care therapy for TAAD remains open surgical repair and after the early medical management en route to the operating room exposure and safe cannulation into the true lumen become the initial concerns for the aortic surgeon. Numerous strategies have been employed over the years. These strategies include peripheral aortic and venous cannulation through the femoral vessels which has been successfully employed since the 1950s (6), right axillary arterial cannulation, first described by Villard in 1976 (7), with either femoral venous or central venous cannulation, transapical cannulation initially described in the 1970s (8) and more recently central cannulation of the aorta with Seldinger technique and transesophageal echocardiography (TEE) guidance to ensure true lumen placement of the aortic cannula. All strategies have merit and must be in the armamentarium of the aortic surgeon. Extensive discussion of the advantages and disadvantages of each cannulation strategy is beyond the scope of this article but the problem solving required to decide on the strategy, and the ability to perform each strategy is a requirement for surgeons who treat aortic dissections.

Management of the aortic root in TAAD can range from replacement with prosthetic valve and graft, to resuspension of a normal valve, to multiple more complex valve sparing procedures involving remodeling or reimplantation of the valve or the Florida Sleeve. As will be discussed in the various review articles included in this series the decision on the extent of repair or the decision to replace the root depends on several aspects of the case; namely if the tear extends into the root, the condition of the valve leaflets, and if there is aneurysmal disease of the root. Valve sparing root replacement with the reimplantation 
technique as initially described by Dr. Tirone David (9) is discussed by the University of Pennsylvania group and reviews indications, technique and special considerations for patients with a bicuspid aortic valve (BAV) (10). A remodeling technique has also been described and appears to be more appropriate for older patients without any history of connective tissue disease with a normal aortic annulus and isolated sinus aneurysmal disease but could be applied to aortic dissection in selected patients (11).

The success of aortic valve sparing techniques coupled with relatively widespread reluctance of surgeons at low volume centers to perform valve-sparing operations led to the innovation that produced the Florida Sleeve technique (12). The Florida Sleeve relies on aortic valve and sinus preservation with an external dacron sleeve that encases the root and allows for sinus expansion while addressing aortic annulus ectasia with an aortic annuloplasty as well as reconstruction of the sinotubular junction without the complexity of the coronary button reimplantation and the multiple aorto-commissural suture lines of the reimplantation and remodeling technique. The Florida Sleeve also can be a particularly useful technique when aberrant coronary anatomy is present or in emergency scenarios, such as aortic dissection, when time is of the essence. The group from the University of Florida where the Florida Sleeve technique originated discuss their technique in the treatment of aortic dissection in this review series.

The extent of resection of the aortic arch in aortic dissection can range from hemiarch reconstruction to total arch reconstruction involving any or all of the brachiocephalic vessels and stabilization of the proximal descending thoracic aorta with a frozen elephant trunk (13). Any aortic arch reconstruction requires lower body circulatory arrest with cerebral protection. In the initial management of aortic arch disease cerebral protection consisted mainly of deep hypothermic cerebral circulatory arrest (DHCA) but over time cerebral perfusion strategies have relegated total body and specifically cerebral circulatory arrest to historical notes as lower body circulatory arrest with either antegrade cerebral perfusion or retrograde cerebral perfusion becoming the standard at most high volume aortic centers (14). Acute TAAD (aTAAD) carries even higher risk of stroke and cerebral malperfusion than elective arch procedures, which makes cerebral protection a particularly important consideration in this circumstance (15). In this review series the group from the University of Colorado review contemporary strategies for cerebral protection in aTAAD including preoperative patient evaluation, perfusion and temperature management, extent of aortic repair, utility of cerebral oximetry and neuromonitoring, and pharmacological adjuncts.

The decision as to the extent of aortic arch reconstruction can be difficult given the significantly longer operative time required for total arch reconstruction in the emergent scenario of a TAAD. The group from the University of Pittsburgh present their experience with hemiarch reconstruction and provide four indications for total arch reconstruction; arch aneurysm, intimal tear extending into the arch, circumferential aortic arch dissection and cerebral malperfusion with dissection extending into either carotid artery. The group from Massachusetts General Hospital and Harvard University also review total arch reconstruction in TAAD and summarize early and late outcomes as well as their specific indications for total arch reconstruction. They present their techniques as well as the long-term prognosis of these patients. The development of stented aortic grafts has led to their utilization in TAAD repair to stabilize the distal arch and descending thoracic aorta in an effort to mitigate the aortic degeneration caused by the residual dissection. The ability to deploy an antegrade stent graft at the time of the open repair allows for direct suturing of the proximal graft to the aorta eliminating the possibility of a type 1 endoleak and also promotes aortic remodeling of the proximal descending thoracic aorta as well as providing a frame for future open or endovascular procedures (16). In this series the group from Yale discuss their experience with antegrade stent grafts in this population and review sizing of the grafts, complications and published results.

Dissections of the descending thoracic aorta, type B aortic dissections (TBAD), can develop as an isolated condition or can be present as a residual dissection after treatment of TAAD. The management and prognosis of TBAD differs significantly from TAAD. Most patients with acute type B dissections (aTBAD) present with back pain or pain related to malperfusion of the spine, viscera or lower extremities. Approximately $20-30 \%$ of patients with type B dissection present with malperfusion of a vascular bed and require urgent intervention. Acute indications for intervention include imminent or actual rupture, pain despite medical management or malperfusion syndrome of any of the previously mentioned vascular beds. Indications for intervening on a chronic type B dissection (cTBAD) include aneurysmal expansion of the involved aorta or drug resistant hypertension.

Traditional open surgical repair for TBAD and descending thoracic aortic aneurysms had been the standard of care yet 
the high morbidity and mortality of the open operation coupled with the lower morbidity, mortality and long-term results of endovascular techniques has led to increasing utilization of thoracic endovascular aortic repair (TEVAR) for treatment of the descending thoracic aorta. Endovascular repair has not yet been proven to be a durable option in young patients with genetic aorotopathies and in many complex situations where the visceral vessels must be addressed endovascular techniques may not be amenable to an acceptable result. Open repair must remain in the armamentarium of an aortic surgeon. The group from the University of Indiana, with an over 20-year experience in open management of cTBAD repair with deep hypothermia and circulatory arrest, present their indications and patient selection, perfusion strategies, surgical techniques and post-operative management principles. The group reports impressive results and provide needed guidance with this approach.

The significant morbidity and mortality associated with open descending thoracic aortic repair led to the development of less invasive endovascular techniques and since the first implantation of a thoracic aortic stent graft was published by Dake in 1994 there has been significant development in the field of (TEVAR) (17). The TEVAR technique involves placement of covered endograft in the descending thoracic aorta. The same principles of open repair have also been addressed with TEVAR including restoration of true lumen flow and exclusion of the intimal tear. Although the endovascular approach is less invasive it is important to note that several important morbidities are not mitigated by the less invasive technique, most importantly spinal cord ischemia. In this series the group from Emory University summarizes short- and long-term outcomes of TEVAR in complicated aTBAD and also review the possible advantages for future management of uncomplicated aTBAD with TEVAR. The advantages of artic remodeling with TEVAR even in uncomplicated aTBAD when combined with strategies to minimize spinal ischemia, like the provisional extension to induce complete attachment (PETTICOAT) approach, selective lumbar drain placement and permissive hypertension, provide impetus for the use of endovascular grafts in uncomplicated aTBAD $(18,19)$.

In complicated aTBAD perfusion to the viscera and or lower extremities is compromised and emergent repair is indicated due to malperfusion. The most simple situation is a single proximal intimal tear which, once covered by a stent graft, allows for restoration of flow through the true lumen and eliminates malperfusion. If secondary tears are present and the malperfusion persists after coverage of the primary tear several surgical and endovascular adjuncts must be considered. These surgical and endovascular adjuncts are presented with accompanying data by the vascular surgery group at the University of Pittsburgh and include visceral branch stenting, dissection flap fenestration and extra-anatomic bypass. The University of Michigan group, since their initial publication in 1997 have challenged the dogma that TAAD needs to be repaired initially with interventions to relieve malperfusion secondarily, if they persist after repair of the TAAD (20). Endovascular therapy for cTBAD had traditionally not been considered as a primary intervention except for in only the highest risk patients $(21,22)$. The thick dissection flap, lack of optimal landing zones and persistent false lumen flow had discouraged many from pursuing endovascular therapy in cTBAD. In this review series the University of Michigan group reviews evidence that TEVAR is a safe and successful therapy in patients too high risk for open repair of CTBAD and describe published techniques and their own institutional approaches to TEVAR in cTBAD. The management of malperfusion will continue to evolve and it is important that ongoing research helps to lead the medical community to an evidence-based approach that optimizes patient outcomes.

Doctor William Osler is credited with the quote, "there is no disease more conducive to clinical bumility than aneurysm of the aorta" yet many would argue that he was in fact incorrect and that thoracic aortic dissection, even more so than aortic aneurysm, has the ability to humble even the most experienced and skilled physician. The disease process of aortic dissection is quite simple to understand yet its treatment remains more complex than ever. Open operations involving cardiopulmonary bypass and deep hypothermic circulatory arrest have in some circumstances given way to percutaneous therapy. This series of reviews in this edition of $7 \mathrm{OVS}$ set out to provide background context, preoperative considerations and expert surgical and interventional techniques in the complex management of aortic dissection. Each review approaches a different aspect of aortic dissection from the aortic annulus into the abdominal aorta. Much time has been spent by each group to provide thoughtful insight and invaluable experience in combating this humbling disease.

\section{Acknowledgments}

Funding: None. 


\section{Footnote}

Provenance and Peer Review: This article was commissioned by the editorial office, fournal of Visualized Surgery for the series "Advancement in the Surgical Treatment of Aortic Dissection". The article did not undergo external peer review.

Conflicts of Interest: All authors have completed the ICMJE uniform disclosure form (available at https://jovs.amegroups.com/ article/view/10.21037/jovs-2020-22/coif). The series "Advancement in the Surgical Treatment of Aortic Dissection" was commissioned by the editorial office without any funding or sponsorship. GJA and IS served as the unpaid Guest Editors of the series. IS serves as an unpaid editorial board member of fournal of Visualized Surgery from September 2018 to August 2020. The authors have no other conflicts of interest to declare.

Ethical Statement: The authors are accountable for all aspects of the work in ensuring that questions related to the accuracy or integrity of any part of the work are appropriately investigated and resolved.

Open Access Statement: This is an Open Access article distributed in accordance with the Creative Commons AttributionNonCommercial-NoDerivs 4.0 International License (CC BY-NC-ND 4.0), which permits the non-commercial replication and distribution of the article with the strict proviso that no changes or edits are made and the original work is properly cited (including links to both the formal publication through the relevant DOI and the license). See: https://creativecommons.org/ licenses/by-nc-nd/4.0/.

\section{References}

1. Sennertus D. Cap. 42. Op Omn Lib 1650;5:306-15.

2. Coady MA, Rizzo JA, Goldstein LJ, et al. Natural history, pathogenesis, and etiology of thoracic aortic aneurysms and dissections. Cardiol Clin 1999;17:615-35; vii.

3. Brown JA, Arnaoutakis GJ, Kilic A, et al. Current trends in the management of acute type A aortic intramural hematoma. J Card Surg 2020;35:2331-7.

4. Sultan I, McGarvey J, Vallabhajosyula P, et al. Routine use of hemiarch during acute type A aortic dissection repair. Ann Cardiothorac Surg 2016;5:245-7.

5. Vallabhajosyula P, Gottret JP, Menon R, et al. Central Repair with Antegrade TEVAR for Malperfusion Syndromes in Acute Debakey I Aortic Dissection. Ann Thorac Surg 2017;103:748-55.

6. Lillehei CW, Cardozo RH. Use of median sternotomy with femoral artery cannulation in open cardiac surgery. Surg Gynecol Obstet 1959;108:706-14.

7. Villard J, Froment JC, Milleret R, et al. Dissection aortique aiguë totale de type I. Intérêt de la perfusion artérielle par voie axillaire [Type I, complete, acute aortic dissection. Value of arterial perfusion by the axillary route (author's transl)]. Ann Chir Thorac Cardiovasc 1976;15:133-5.

8. Zwart HH, Kralios A, Kwan-Gett CS, et al. First clinical application of transarterial closed-chest left ventricular (TaCLV) bypass. Trans Am Soc Artif Intern Organs 1970;16:386-91.

9. David TE, Feindel CM. An aortic valve-sparing operation for patients with aortic incompetence and aneurysm of the ascending aorta. J Thorac Cardiovasc Surg 1992;103:617-21; discussion 622.

10. Sultan I, Komlo CM, Bavaria JE. How I Teach a Valve-Sparing Root Replacement. Ann Thorac Surg 2016;101:422-5.

11. David TE. Aortic valve sparing operations. Semin Thorac Cardiovasc Surg 2011;23:146-8.

12. Hess PJ Jr, Klodell CT, Beaver TM, et al. The Florida sleeve: a new technique for aortic root remodeling with preservation of the aortic valve and sinuses. Ann Thorac Surg 2005;80:748-50.

13. Sultan I, Szeto WY. Decision making in acute DeBakey I aortic dissection: Balancing extensive arch reconstruction versus mortality. J Thorac Cardiovasc Surg 2016;151:349-50.

14. Arnaoutakis GJ, Vallabhajosyula P, Bavaria JE, et al. The Impact of Deep Versus Moderate Hypothermia on Postoperative Kidney Function After Elective Aortic Hemiarch Repair. Ann Thorac Surg 2016;102:1313-21. 
15. Sultan I, Bianco V, Patel HJ, et al. Surgery for type A aortic dissection in patients with cerebral malperfusion: Results from the International Registry of Acute Aortic Dissection. J Thorac Cardiovasc Surg 2019. [Epub ahead of print]. doi:10.1016/ j.jtcvs.2019.11.003.

16. Sultan I, Wallen TJ, Habertheuer A, et al. Concomitant antegrade stent grafting of the descending thoracic aorta during transverse hemiarch reconstruction for acute DeBakey I aortic dissection repair improves aortic remodeling. J Card Surg 2017;32:581-92.

17. Dake MD, Miller DC, Semba CP, et al. Transluminal placement of endovascular stent-grafts for the treatment of descending thoracic aortic aneurysms. N Engl J Med 1994;331:1729-34.

18. Sultan I, Dufendach K, Kilic A, et al. Bare Metal Stent Use in Type B Aortic Dissection May Offer Positive Remodeling for the Distal Aorta. Ann Thorac Surg 2018;106:1364-70.

19. Grimm JC, Sultan I. Mitigating spinal cord ischemia in thoracic endovascular aortic repair: is CSF drainage mandatory? Ann Thorac Surg 2020;110:1474-5.

20. Deeb GM, Williams DM, Quint LE, et al. Surgical delay for acute type A dissection with malperfusion. Ann Thorac Surg 1997;64:1669-75; discussion 1675-7.

21. Sultan I, Siki MA, Bavaria JE, et al. Predicting Distal Aortic Remodeling After Endovascular Repair for Chronic DeBakey III Aortic Dissection. Ann Thorac Surg 2018;105:1691-6.

22. Bianco V, Sultan I, Kilic A, et al. Concomitant left subclavian artery revascularization with carotid-subclavian transposition during zone 2 thoracic endovascular aortic repair. J Thorac Cardiovasc Surg 2020;159:1222-7.

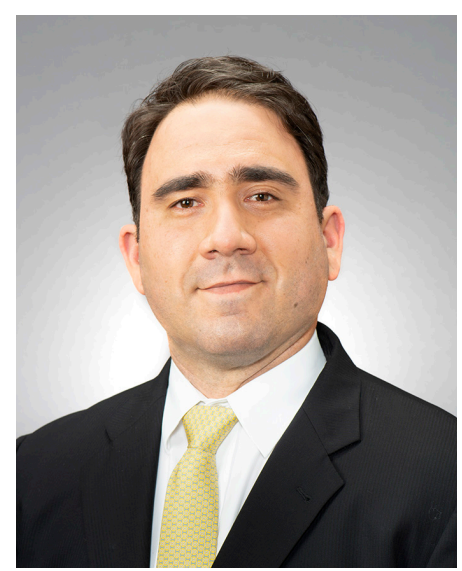

Derek Serna-Gallegos

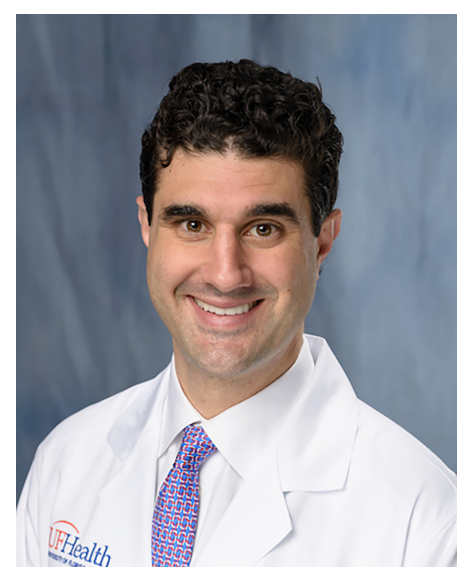

George J. Arnaoutakis

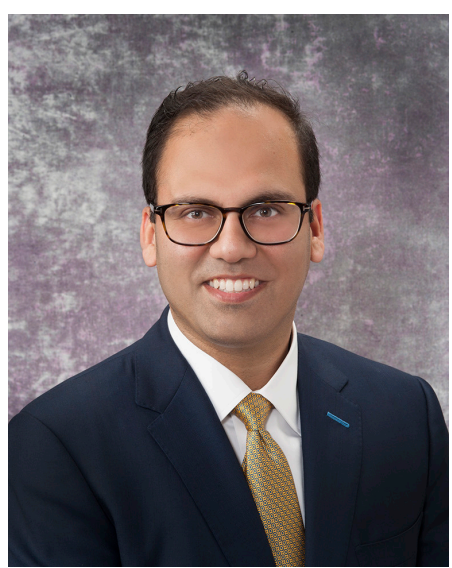

Ibrahim Sultan 


\section{Derek Serna-Gallegos ${ }^{1,2}$, George J. Arnaoutakis ${ }^{3}$, Ibrahim Sultan}

${ }^{1}$ Division of Cardiac Surgery, Department of Cardiothoracic Surgery, University of Pittsburgh, Pittsburgh, PA, USA; ${ }^{2}$ Heart and Vascular Institute, University of Pittsburgh Medical Center, Pittsburgh, PA, USA; ${ }^{3}$ Division of Thoracic and Cardiovascular Surgery,

Department of Surgery, University of Florida, Gainesville, Florida, USA.

(Email: Sernagallegosdr@upmc.edu; george.arnaoutakis@surgery.ufl.edu; sultani@upmc.edu)

Received: 18 July 2020; Accepted: 29 July 2020; Published: 20 January 2021. doi: 10.21037 /jovs-2020-22

View this article at: http://dx.doi.org/10.21037/jovs-2020-22

doi: $10.21037 /$ jovs-2020-22

Cite this article as: Serna-Gallegos D, Arnaoutakis GJ, Sultan I. Advancements in the management of thoracic aortic dissection. J Vis Surg 2021;7:2. 